\title{
A modified infeasible homotopy algorithm for computing fixed point in general non-convex set
}

\author{
Zhichuan Zhu ${ }^{\mathrm{a}, *}$, Ruifeng Wu ${ }^{\mathrm{b}}$, Yanchun Xing ${ }^{\mathrm{a}}$ \\ a School of Statistics, Jilin University of Finance and Economics, Changchun, Jilin 130117, China. \\ ${ }^{b}$ School of Applied Mathematics, Jilin University of Finance and Economics, Changchun, Jilin 130117, China. \\ Communicated by C. Alaca
}

\begin{abstract}
In this paper, to find a fixed point of self-mapping in the general non-convex set with both equality constraints and inequality constraints, a modified infeasible homotopy for perturbing only inequality constraints is constructed and the global convergence of the smooth homotopy pathways is proved under some much weaker conditions. The advantage of the modified homotopy is that the initial point needs to be only in the shifted set with only inequality constraints, not necessarily, a feasible point in the original set, and hence it is more convenient to be implemented than the existing methods. The feasibility and effectiveness of the modified homotopy method is shown by some numerical tests. (c)2017 All rights reserved.
\end{abstract}

Keywords: Infeasible homotopy, fixed point, self-mapping, non-convex set.

2010 MSC: 47H10, 55M20.

\section{Introduction}

Fixed point theory has been broadly implemented in nonlinear analysis, integral and differential equations, dynamical system theory, game theory, optimization problems and other fields. Recently, lots of results on the fixed point theory and algorithms have appeared and attracted many attentions, see references [2, 3, 6, 8, 12-16]. Among all of the fixed point theories, the famous Brouwer fixed point theorem only requires that the self-mapping $\phi: \Omega \rightarrow \Omega$ is continuous without requiring any monotone condition and has been extensively applied in game theory, equilibrium problems and other across numerous fields of mathematics. For computing the Brouwer fixed point of a self-mapping in a convex set, Kellogg et al. [7] constructed a single homotopy and gave an innovative proof in 1976. In 1978, to compute the Brouwer fixed point more conveniently in convex set, Chow et al. [4] constructed a more simple fixed point homotopy.

In 1996, to numerically compute a fixed point of a twice continuous differentiable self-mapping in a set $\Omega=\left\{x: g_{i}(x) \leqslant 0, i=1,2, \cdots, m\right\}$ without any convexity condition, Yu and Lin [17] first proposed

\footnotetext{
*Corresponding author

Email addresses: zhuzcnh@126.com (Zhichuan Zhu), wuruifeng@jlufe.edu.cn (Ruifeng Wu), xingyanchun778@163.com (Yanchun Xing)
}

doi:10.22436/jnsa.010.11.27 
an interior point combined homotopy, which was consisted of a fixed point homotopy and a Newton homotopy, as follows:

$$
H(w, t)=\left(\begin{array}{c}
(1-t)\left(x-\phi(x)+\sum_{i=1}^{m} \nabla g_{i}(x) y_{i}\right)+t\left(x-x^{0}\right) \\
Y g(x)-t Y^{0} g\left(x^{0}\right)
\end{array}\right),
$$

where $\left(x^{0}, y^{0}\right) \in \Omega^{0} \times R_{++}^{m}, y_{i} \geqslant 0, t \in[0,1], g(x)=\left(g_{1}(x), \cdots, g_{m}(x)\right)^{\top}, Y$ and $Y^{0}$ denote the diagonal matrices whose $i$-th diagonal element are $y_{i}$ and $y_{i}^{0}$ respectively, and the strict feasible set

$$
X^{0}=\left\{x: g_{i}(x)<0, i=1,2, \cdots, m\right\} .
$$

In 2008, to compute a fixed point of a self-mapping in the general non-convex sets, Su and Liu [9] generalized the homotopy (1.1) to compute fixed point of self-mapping in a broader class of non-convex bounded sets $\Omega=\left\{x: g_{i}(x) \leqslant 0, h_{j}(x)=0, i=1,2, \cdots, m, j=1,2, \cdots, l\right\}$ as follows:

$$
H(w, t)=\left(\begin{array}{c}
(1-t)\left(x-\phi(x)+\sum_{i=1}^{m} \nabla g_{i}(x) y_{i}\right)+\sum_{j=1}^{l} \nabla h_{j}(x) z_{j}+t\left(x-x^{0}\right) \\
h(x) \\
Y g(x)-t Y^{0} g\left(x^{0}\right)
\end{array}\right),
$$

where $w=(x, y, z) \in R^{n} \times R_{+}^{m} \times R^{l},\left(x^{0}, y^{0}\right) \in X^{0} \times R_{++}^{m}, t \in[0,1], h(x)=\left(h_{1}(x), h_{2}(x), \cdots, h_{l}(x)\right)^{\top}$, and the strict feasible set $\Omega^{0}=\left\{x: g_{i}(x)<0, h_{j}(x)=0, i=1,2, \cdots, m, j=1,2, \cdots, l\right\}$.

In 2013, to relax the boundedness condition and weaken the normal cone condition in references [9, 17], Zhu et al. [20] constructed a modified combined homotopy for computing fixed point of a selfmapping in the general unbounded non-convex sets $\Omega=\left\{x: g_{i}(x) \leqslant 0, h_{j}(x)=0, i=1,2, \cdots, m, j=\right.$ $1,2, \cdots, l\}$ as follows:

$$
H(w, t)=\left(\begin{array}{c}
(1-t)\left(x-\phi(x)+\sum_{i=1}^{m} \eta_{i}\left(x, y_{i}\right)\right)+\sum_{j=1}^{l} \zeta_{j}\left(x, z_{j}\right)+t\left(x-x^{(0)}\right) \\
h(x) \\
Y g(x)-t Y^{(0)} g\left(x^{(0)}\right)
\end{array}\right)
$$

where $w=(x, y, z), w^{(0)}=\left(x^{(0)}, y^{(0)}, z^{(0)}\right), y=\left(y_{1}, \cdots, y_{m}\right)^{\top}, z=\left(z_{1}, \cdots, z_{l}\right), Y=\operatorname{diag}(y), \eta_{i}\left(x, y_{i}\right)$ and $\zeta_{\mathfrak{j}}\left(x, z_{j}\right)$ are the so-called hair mappings. The existence and global convergence of the smooth homotopy pathway was also proved under much weaker pseudo cone condition.

Since the interior point combined homotopy method requires that the initial point must be chosen in the original feasible set, to enlarge the chosen scope of initial points, in 2011, Su et al. [11] presented a boundary perturbation homotopy method on non-convex bounded sets with only inequality constraints. In 2015, Su and Qian [10] constructed a modified combined homotopy method by a perturbation on the inequality constraints of the general non-convex unbounded sets. In 2015, Fan et al. [5] proposed an infeasible interior point homotopy method for enlarging the choice scope of initial points by a perturbation on equality constraints on the general unbounded sets, but some conditions were lost in the proof of the global convergence. In 2017, Zhu et al. [22] proposed a constraint set swelling homotopy method for computing the fixed point of self-mapping on the general non-convex set.

For the general non-convex sets with both inequality constraints and equality constraints, to enlarge the chosen scope of initial points and weaken the convergent conditions, it seems reasonable to construct the homotopy directly as follows:

$$
H(w, t)=\left(\begin{array}{c}
(1-t)\left(x-\phi(x)+\sum_{i=1}^{m} \nabla g_{i}(x) y_{i}\right)+\sum_{j=1}^{l} \nabla h_{j}(x) z_{j}+t\left(x-x^{0}\right) \\
h(x)-t h\left(x^{0}\right) \\
Y g(x)-t Y^{0} g\left(x^{0}\right)
\end{array}\right) .
$$


However, the probability-one regularity of the homotopy equation (1.2) cannot be assured, since for all $t \in(0,1]$, the following sub-matrix may be not full row rank by taking $x^{0}$ and $y^{0}$ as variate

$$
\frac{\partial \mathrm{H}\left(x, x^{0}, y^{0}, \mathrm{t}\right)}{\partial\left(x, x^{0}, y^{0}\right)}=\left(\begin{array}{ccc}
\Xi & -\mathrm{tI} & 0 \\
(\nabla h(x))^{\top} & -\mathrm{t}\left(\nabla h\left(x^{0}\right)\right)^{\top} & 0 \\
Y(\nabla g(x))^{\top} & -\mathrm{t} Y^{0}\left(\nabla \mathrm{g}\left(x^{0}\right)\right)^{\top} & -\operatorname{tdiag}\left(g\left(x^{0}\right)\right)
\end{array}\right),
$$

where $\Xi=(1-t)\left(I-\nabla \phi(x)+\sum_{i=1}^{m} \nabla^{2} g_{i}(x) y_{i}\right)+\sum_{j=1}^{l} \nabla^{2} h_{j}(x) z_{j}+t I$, and $\operatorname{diag}\left(g\left(x^{0}\right)\right)$ denotes the diagonal matrix with its $i$-th diagonal element $g_{i}\left(x^{0}\right)$.

In this paper, to overcome the defects of the initial points which must be in the original feasible set and weaken the convergent conditions, a modified infeasible homotopy, which is more convenient and practical, for computing the fixed point of self-mapping on the general non-convex sets is constructed and the existence and global convergence of the smooth homotopy pathways is proven under some mild conditions.

The rest of the paper is organized as follows. In Section 2, an equivalent condition of the existence of fixed point will be given and some lemmas which will be used for proving the main result will be presented. In Section 3, a modified infeasible homotopy equation for computing the fixed point on the general non-convex sets is constructed and the existence and global convergence of a smooth path from any initial point in constraint shifting set to a fixed point of any twice continuous differentiable selfmapping will be proved. In Section 4, some numerical examples will be given to show the feasibility and effectiveness of the proposed method.

\section{Preliminaries}

Throughout the paper, the general non-convex closed subset $\Omega \in \mathrm{R}^{\mathrm{n}}$ is defined as follows:

$$
\Omega=\left\{x \in R^{n}: g_{i}(x) \leqslant 0, h_{j}(x)=0, i=1,2, \cdots, m, j=1,2, \cdots, l\right\} .
$$

In this paper, to enlarge the chosen scope of initial points and weaken the convergent conditions, a shifted constraint function is constructed as $\hat{g}_{i}(x, t), i=1,2, \cdots, m$, which satisfies

$$
\hat{g}_{i}(x, 0)=g_{i}(x), \quad i=1,2, \cdots, m .
$$

For the sake of convenience, denote

$$
\begin{aligned}
\hat{\Omega} & =\left\{x: g_{i}(x) \leqslant 0, i=1, \cdots, m\right\}, \\
\hat{\Omega}(t) & =\left\{x: \hat{g}_{i}(x, t) \leqslant 0, i=1, \cdots, m\right\}, \\
\hat{\Omega}^{0}(t) & =\left\{x: \hat{g}_{i}(x, t)<0, i=1, \cdots, m\right\}, \\
\partial \hat{\Omega}(t) & =\hat{\Omega}(t) \backslash \hat{\Omega}^{0}(t),
\end{aligned}
$$

and

$$
\mathbb{I}_{t}(x)=\left\{i \in\{1, \cdots, m\}: \hat{g}_{\mathfrak{i}}(x, t)=0\right\},
$$

respectively. Let $\nabla \hat{g}(x, t)$ denote the gradient of $\hat{g}(x, t)$ with respect to the variable $x$.

To prove the main results, the shifted constraint function $\hat{g}(x, t)$ and equality constraint function $h(x)$ must simultaneously satisfy the following conditions.

(A1) $\hat{g}_{i}(x, t), i=1,2, \cdots, m$ and $h_{j}(x), j=1,2, \cdots, l$ are three times continuous differentiable.

(A2) For all $t \in[0,1], \hat{\Omega}^{0}(t) \neq \phi$ and $\tilde{\Omega}=\cup_{t \in[0,1]} \hat{\Omega}(t)$ is bounded. 
(A3) For all $t \in[0,1]$, the matrix $\left\{\left(\nabla \hat{g}_{i}(x, t)\right)_{i \in \mathbb{I}_{t}(x)}, \nabla h(x)\right\}$ is positive-linearly independent at $x \in \partial \hat{\Omega}(t)$, i.e.,

$$
\sum_{i \in \mathbb{I}_{t}(x)} \alpha_{i} \nabla \hat{g}_{i}(x, t)+\sum_{j=1}^{m} \beta_{j} \nabla h_{j}(x)=0, \alpha_{i} \in R_{+}, \beta_{j} \in R \Rightarrow \alpha_{i}=\beta_{j}=0, i \in \mathbb{I}_{t}(x) \text { and } j=1,2, \cdots, m .
$$

(A4) For any $t \in[0,1]$, and for all $x \in \partial \hat{\Omega}(t)$, the normal cone of $\hat{\Omega}(t)$ only meets $\partial \hat{\Omega}(t)$ at $x$, i.e.,

$$
\left\{x+\sum_{i \in \mathbb{I}_{\mathfrak{t}}(x)} \nabla \hat{g}_{i}(x, t) y_{i} \mid y_{i} \geqslant 0\right\} \cap \hat{\Omega}(t)=\{x\}, \quad \forall x \in \partial \hat{\Omega}(t) .
$$

To compute a fixed point of self-mapping in the general non-convex set by the combined homotopy method, the following theorem which is an equivalent condition of the existence for fixed point in the general non-convex set $\Omega$ is important and will be used in the next section.

Theorem 2.1. Let the non-convex set $\Omega$ be defined as (2.1), and the constraint functions $g_{i}(x), i=1,2, \cdots, m$ and $h_{j}(x), j=1,2, \cdots, l$ be three times continuous differentiable. If the conditions (A1)-(A4) hold, then for any twice continuous differentiable self-mapping $\phi: \Omega \rightarrow \Omega, x \in \Omega$ is a fixed point of the mapping $\phi(x)$ if and only if there exists a vector $(y, z) \in R_{+}^{m} \times R^{l}$, such that $(x, y, z)$ is a solution of the following system:

$$
\begin{aligned}
& x-\phi(x)+\nabla g(x) y+\nabla h(x) z=0, \\
& h(x)=0, \\
& Y g(x)=0, \quad g(x) \leqslant 0, \quad y \geqslant 0 .
\end{aligned}
$$

Proof. When the parameter $t=0$, the conditions (A1)-(A3) are the same as the conditions of [9, Lemma 2.1]. Therefore, the proof is omitted here.

To prove the main results, the following parameterized Sard theorem (see [4, Theorem 2.1]) will be used and is presented here. Let $U \subset R^{n}$ be an open set and $\varphi: U \rightarrow R^{p}$ be a $C^{\alpha}(\alpha>\max \{0, n-p\})$ mapping. We say that $y \in R^{p}$ is a regular value for $\varphi$ if

$$
\text { Range }[\partial \varphi(x) / \partial x]=R^{p}, \quad \forall x \in \varphi^{-1}(y) .
$$

Lemma 2.2. Let $\mathrm{V} \subset \mathrm{R}^{\mathrm{n}}, \mathrm{U} \subset \mathrm{R}^{\mathrm{m}}$ be open sets, and let $\varphi: \mathrm{V} \times \mathrm{U} \rightarrow \mathrm{R}^{\mathrm{k}}$ be a $\mathrm{C}^{\alpha}$ mapping, where

$$
\alpha>\max \{0, m-k\} .
$$

If $0 \in \mathrm{R}^{\mathrm{k}}$ is a regular value of $\varphi$, then for almost all $\mathrm{a} \in \mathrm{V}, 0$ is a regular value of $\varphi_{\mathrm{a}}=\varphi(\mathrm{a}, \cdot)$.

\section{Main result}

By Theorem 2.1, to find a fixed point of a twice continuous differentiable self-mapping is equivalent to solve the system (2.2). Hence, to solve the equivalent system (2.2) more conveniently, for any given constant $\eta \in R_{++}^{m}$ and any initial point $x^{0} \in \hat{\Omega}^{0}(1)$, a modified infeasible homotopy equation is constructed as follows:

$$
H(w, t)=\left(\begin{array}{cc}
(1-t)(x-\phi(x)+\nabla \hat{g}(x, t) y+\nabla h(x) z)+t\left(x-x^{0}\right) \\
Y \hat{g}(x, t)+t \eta \\
h(x)-t z
\end{array}\right)=0,
$$

where $w=(x, y, z)^{\top} \in R^{n} \times R_{+}^{m} \times R^{l}, Y=\operatorname{diag}\left(y_{1}, y_{2}, \cdots, y_{m}\right)$, and $\hat{g}(x, t)$ is the shifted constraint function which satisfies conditions (A1)-(A4).

For the homotopy equation (3.1), when $t=0$, the homotopy equation $H(x, 0)=0$ turns to the equivalent system (2.2). When $t=1$, the homotopy equation $H(x, 1)=0$ can be written as follows:

$$
\left(\begin{array}{r}
x-x^{0} \\
\mathrm{Yg}(x, 1)+\eta \\
h(x)-z
\end{array}\right)=0
$$


which has a unique simple solution $(x, y, z)=\left(x^{0}, y^{0}, z^{0}\right)=\left(x^{0},-\left[\operatorname{diag}\left(\hat{g}\left(x^{0}, 1\right)\right)\right]^{-1} \eta, h\left(x^{0}\right)\right)$.

For any given initial point $w^{0} \in \Omega^{0}(1) \times R_{++}^{m} \times R^{l}$, the zero-point set of $H(w, t)=0$ is denoted as follows

$$
\mathrm{H}_{w^{0}}^{-1}(0)=\left\{(w, t) \in \Omega^{0}(1) \times \mathrm{R}_{++}^{\mathrm{m}} \times \mathrm{R}^{\mathrm{l}} \times(0,1]: \mathrm{H}(w, \mathrm{t})=0\right\} .
$$

Theorem 3.1. Suppose the constraint set $\Omega$ is defined as (2.1). If the constraint functions $g_{i}(x), i=1,2, \cdots, m$ and $h_{j}(x), j=1,2, \cdots$, lare all three times continuous differentiable, and conditions (A1)-(A4) hold, then any twice continuous differentiable self-mapping $\phi: \Omega \rightarrow \Omega$ has a fixed point in $\Omega$, and for any $w^{0} \in \hat{\Omega}(1)^{0} \times R_{++}^{m} \times R^{l}$, the zero-point set $\mathrm{H}_{w^{0}}^{-1}(0)$ of the homotopy equation (3.1) contains a smooth curve $\Gamma_{w^{0}} \subset \hat{\Omega}(1) \times \mathrm{R}_{+}^{\mathrm{m}} \times \mathrm{R}^{\mathrm{l}} \times(0,1]$, which begins from $\left(x^{0}, y^{0}, z^{0}, 1\right)$ and terminates in or approaches to the hyperplane $t=0$. Moreover, if $(\tilde{x}, \tilde{y}, \tilde{z}, 0)$ is an ending limit point of the smooth curve $\Gamma_{w^{0}}$, then $\tilde{w}=(\tilde{x}, \tilde{y}, \tilde{z})$ is a solution to system (2.2) and the $\tilde{x}$-component is a fixed point of $\phi(x)$ in $\Omega$.

Proof. Taking $x^{0}$ as variate and let $\widehat{\mathrm{H}}\left(w, x^{0}, \mathrm{t}\right)$ be the same mapping as $\mathrm{H}(w, \mathrm{t})$. Consider the sub-matrix of the Jacobian $\mathrm{D} \widehat{\mathrm{H}}\left(w, x^{0}, \mathrm{t}\right)$ as follows:

$$
\frac{\partial \widehat{\mathrm{H}}\left(w, x^{0}, \mathrm{t}\right)}{\partial\left(x^{0}, y, z\right)}=\left(\begin{array}{ccc}
-\mathrm{tI} & * & * \\
0 & \operatorname{diag}(\hat{g}(x, t)) & 0 \\
0 & 0 & -\mathrm{tI}
\end{array}\right)
$$

For all $t \in(0,1]$, and for any $x \in \hat{\Omega}(1)$, since $\eta \in R_{++}^{m}$ and $Y \hat{g}(x, t)+t \eta=0$, we can obtain that the matrix $\operatorname{diag}(\hat{g}(x, t))$ is nonsingular, which implies that the sub-matrix $\frac{\partial \widehat{H}\left(w, x^{0}, t\right)}{\partial\left(x^{0}, y, z\right)}$ is nonsingular. Hence, the matrix $\mathrm{D} \widehat{\mathrm{H}}\left(w, x^{0}, \mathrm{t}\right)$ is a matrix of full row rank, which means that 0 is a regular value of $\hat{\mathrm{H}}\left(w, x^{0}, \mathrm{t}\right)$. Therefore, by Lemma 2.2, for almost all $x^{0} \in \hat{\Omega}^{0}(1), 0$ is a regular value of $H(w, t)$. For the given $w^{0} \in \hat{\Omega}^{0}(1) \times R_{++}^{m} \times R^{l}$, if 0 is a regular value of $\mathrm{H}(w, \mathrm{t})$, from the fact that $\mathrm{H}\left(w^{0}, 1\right)=0$, the matrix

$$
\frac{\partial \mathrm{H}\left(w^{0}, 1\right)}{\partial w}=\left(\begin{array}{ccc}
\mathrm{I} & 0 & 0 \\
* & \operatorname{diag}\left(\hat{\mathrm{g}}\left(\chi^{0}, 1\right)\right) & 0 \\
* & 0 & -\mathrm{I}
\end{array}\right)
$$

is also nonsingular, and the famous implicit function theorem, we get that $\mathrm{H}_{w^{0}}^{-1}(0)$ must contain a smooth curve $\Gamma_{w^{0}}$, which starts from $\left(x^{0}, y^{0}, z^{0}, 1\right)$, goes into $\Omega^{0}(1) \times R_{++}^{m} \times R^{l} \times(0,1)$ and terminates in the boundary of $\Omega(t) \times R_{+}^{m} \times R^{l} \times[0,1]$.

Let $(\tilde{w}, \tilde{t})$ be an ending limit point of the smooth curve $\Gamma_{w^{0}}$ as $t \rightarrow 0$. Then, only the following five cases may be possible:

(i) $(\tilde{w}, \tilde{\mathrm{t}}) \in \hat{\Omega}(1) \times \mathrm{R}_{+}^{\mathrm{m}} \times \mathrm{R}^{\mathrm{l}} \times\{1\},\|(\tilde{\mathrm{y}}, \tilde{z})\|<\infty$;

(ii) $(\tilde{w}, \tilde{t}) \in \hat{\Omega}(\tilde{\mathrm{t}}) \times \mathrm{R}_{+}^{\mathrm{m}} \times \mathrm{R}^{\mathrm{l}} \times[0,1],\|(\tilde{\mathrm{y}}, \tilde{z})\|=\infty$;

(iii) $(\tilde{w}, \tilde{\mathrm{t}}) \in \hat{\Omega}(\tilde{\mathrm{t}}) \times \partial R_{+}^{\mathrm{m}} \times \mathrm{R}^{\mathrm{l}} \times(0,1),\|(\tilde{\mathrm{y}}, \tilde{z})\|<\infty$;

(iv) $(\tilde{w}, \tilde{\mathrm{t}}) \in \partial \hat{\Omega}(\tilde{\mathrm{t}}) \times R_{++}^{\mathrm{m}} \times \mathrm{R}^{\mathrm{l}} \times(0,1),\|(\tilde{\mathrm{y}}, \tilde{z})\|<\infty$;

(v) $(\tilde{w}, \tilde{\mathrm{t}}) \in \hat{\Omega} \times R_{+}^{\mathrm{m}} \times \mathrm{R}^{\mathrm{l}} \times\{0\},\|(\tilde{\mathrm{y}}, \tilde{z})\|<\infty$.

Since the homotopy equation $\mathrm{H}(w, 1)=0$ has a unique simple solution

$$
w^{0}=\left(x^{0}, y^{0}, z^{0}\right) \text { in } \hat{\Omega}^{0}(1) \times R_{++}^{m} \times R^{l},
$$

and $\frac{\partial H\left(w^{0}, 1\right)}{\partial w}$ is nonsingular, case (i) is impossible.

Next, we will prove that case (ii) is also impossible. If the case (ii) holds, there must exist a sequence of points $\left\{\left(x^{k}, y^{k}, z^{k}, t^{k}\right)\right\} \subset \Gamma_{w^{0}}$ such that $x^{k} \rightarrow \tilde{x} \in \hat{\Omega}(\tilde{t}),\left\|\left(y^{k}, z^{k}\right)\right\| \rightarrow \infty$, and $t^{k} \rightarrow \tilde{t} \in[0,1]$ as $k \rightarrow \infty$. From the first equation of the homotopy equation (3.1), we can get

$$
\left(1-t^{k}\right)\left(x^{k}-\phi\left(x^{k}\right)+\sum_{i=1}^{m} y_{i}^{k} \nabla \hat{g}_{i}\left(x^{k}, t^{k}\right)+\sum_{j=1}^{l} \nabla h_{j}\left(x^{k}\right) z_{j}^{k}\right)+t^{k}\left(x^{k}-x^{0}\right)=0 .
$$

When $k \rightarrow \infty$, only the following three subcases are possible: 
(I) $\tilde{\mathrm{t}}=1$;

(II) $\tilde{\mathrm{t}} \in(0,1)$;

(III) $\tilde{\mathrm{t}}=0$.

(I) $\tilde{\mathrm{t}}=1$. From the third equation of (3.1), we have $z^{k} \rightarrow h(\tilde{x})$ as $k \rightarrow \infty$, which implies that $\left\{z^{k}\right\}$ is bounded. Hence, the sequence of points $\left\|y^{k}\right\| \rightarrow \infty$. Then, by the second equation of (3.1) and $\left\|y^{k}\right\| \rightarrow \infty$, we get $\tilde{x} \in \partial \hat{\Omega}(1)$.

If $\left\|\left(1-t_{k}\right) y^{k}\right\|$ is bounded, without loss of generality, suppose that $\left(1-t_{k}\right) y^{k} \rightarrow \tilde{y}$, then $\tilde{y}_{i}=0$ for $i \notin \mathbb{I}_{1}(\tilde{x})$ by the second equation of (3.1). Now, taking limits in (3.2) as $k \rightarrow \infty$, by the fact that $\left\{z^{k}\right\}$ is bounded and conditions (A1) and (A2) hold, we have

$$
\begin{aligned}
x^{0}= & \tilde{x}+\lim _{k \rightarrow \infty}\left(1-t_{k}\right)\left(x^{k}-\phi\left(x^{k}\right)+\sum_{i=1}^{m} \nabla \hat{g}_{i}\left(x^{k}, t_{k}\right) y_{i}^{k}+\sum_{j=1}^{l} \nabla h_{j}\left(x^{k}\right) z_{j}^{k}\right) \\
= & \tilde{x}+\lim _{k \rightarrow \infty}\left(1-t_{k}\right)\left(x^{k}-\phi\left(x^{k}\right)+\sum_{i \notin \mathbb{I}_{1}(\tilde{x})} \nabla \hat{g}_{i}\left(x^{k}, t_{k}\right) y_{i}^{k}+\sum_{j=1}^{l} \nabla h_{j}\left(x^{k}\right) z_{j}^{k}\right) \\
& +\lim _{k \rightarrow \infty} \sum_{i \in \mathbb{I}_{1}(\tilde{x})}\left(1-t_{k}\right) y_{i}^{k} \nabla \hat{g}_{i}\left(x^{k}, t_{k}\right) \\
= & \tilde{x}+(1-\tilde{t})\left(\tilde{x}-\phi(\tilde{x})+\sum_{j=1}^{m} \nabla h_{j}(\tilde{x}) \tilde{z}_{j}\right)+\sum_{i \notin \mathbb{I}_{1}(\tilde{x})} \nabla \hat{g}_{i}(\tilde{x}, \tilde{t}) \tilde{y}_{i} \\
& +\sum_{i \in \mathbb{I}_{1}(\tilde{x})} \tilde{y}_{i} \nabla \hat{g}_{i}(\tilde{x}, \tilde{t}) \\
= & \tilde{x}+\sum_{i \in \mathbb{I}_{1}(\tilde{x})} \tilde{y}_{i} \nabla \hat{g}_{i}(\tilde{x}, 1),
\end{aligned}
$$

which contradicts with the condition (A4).

If $\left\|\left(1-t^{k}\right) y^{k}\right\|$ is unbounded, the proof is the same as the following case (II).

(II) $\tilde{t} \in(0,1)$. From the third equation of (3.1), we have $z^{k} \rightarrow h(\tilde{x}) / \tilde{t}$ as $k \rightarrow \infty$, which implies that $\left\{z^{k}\right\}$ is bounded. Hence, only the sequence of points $\left\|y^{k}\right\| \rightarrow \infty$.

Since $\tilde{t} \in(0,1)$ and $\left\|y^{k}\right\| \rightarrow \infty,\left(1-t_{k}\right) y^{k}$ is unbounded, without loss of generality, we assume that $\left(1-t_{k}\right) y^{k} /\left\|\left(1-t_{k}\right) y^{k}\right\| \rightarrow \alpha^{*}$ with $\left\|\alpha^{*}\right\|=1$ and $\alpha_{i}^{*}=0$ for $i \notin \mathbb{I}_{\tilde{\mathfrak{t}}}(\tilde{x})$. Dividing both sides of the equation (3.2) by $\left\|\left(1-t_{k}\right) y^{k}\right\|$ and taking limits as $k \rightarrow \infty$, we can get

$$
\sum_{i \in \mathbb{I}_{\mathfrak{t}}(\tilde{x})} \alpha_{i}^{*} \nabla \hat{g}_{\mathfrak{i}}(\tilde{x}, \tilde{\mathfrak{t}})=0
$$

which contradicts with condition (A3).

(III) $\tilde{\mathrm{t}}=0$. Without loss of generality, suppose that $\left(y^{k}, z^{k}\right) /\left\|\left(y^{k}, z^{k}\right)\right\| \rightarrow\left(\alpha^{*}, \beta^{*}\right)$ with $\left\|\left(\alpha^{*}, \beta^{*}\right)\right\|=1$ and $\alpha_{i}^{*}=0$, for $i \notin \mathbb{I}_{0}(\tilde{x})$. Then, through dividing the both sides of equation (3.2) by $\left\|\left(y^{k}, z^{k}\right)\right\|$ and taking limits as $k \rightarrow \infty$, we have

$$
\sum_{i \in \mathbb{I}_{0}(\tilde{x})} \alpha_{i}^{*} \nabla \hat{g}_{i}(\tilde{x}, 0)+\sum_{j=1}^{m} \beta_{j}^{*} \nabla h_{j}(\tilde{x})=0,
$$

which contradicts condition (A3).

Concluded from the above discussions on subcases (I)-(III), we get that case (ii) is also impossible.

Now, we prove that case (iii) and case (iv) are also impossible. From the second equation of (3.1) $\operatorname{diag}(\hat{\mathrm{g}}(\tilde{\mathrm{x}}, \tilde{\mathrm{t}})) \tilde{\mathrm{y}}+\tilde{\mathrm{t}} \eta=0$, we have that $\tilde{\mathrm{t}}>0$ and $\tilde{y} \in \partial R_{+}^{\mathrm{m}}$, i.e., $\tilde{y}_{i}=0$ for some $1 \leqslant i \leqslant m$ cannot 
happen simultaneously. Hence, case (iii) is also impossible. If $\tilde{y}>0$ and $\tilde{t}>0$, from the equation $\operatorname{diag}(\hat{\mathrm{g}}(\tilde{\mathrm{x}}, \tilde{\mathrm{t}})) \tilde{\mathrm{y}}+\tilde{\mathrm{t}} \eta=0$, we get $\operatorname{diag}(\hat{\mathrm{g}}(\tilde{\mathrm{x}}, \tilde{\mathrm{t}}))<0$, which implies that the case (iv) is impossible too.

In conclusion, from the above discussions, the case $(\mathrm{v})$ is the only possible case. Hence, $\Gamma_{w^{0}}$ must terminate in or approach to the hyperplane at $\tilde{t}=0$ and $\tilde{w}=(\tilde{x}, \tilde{y}, \tilde{z})$ is a solution to the system (2.2). By Theorem 2.1, we have that $\tilde{x}$ is a fixed point of $\phi(x)$ in $\Omega$.

The proof is complete.

\section{Numerical test}

In this section, to show the feasibility and effectiveness of the modified infeasible homotopy method, some numerical tests will be done through numerically tracing the smooth curve $\Gamma_{w^{0}}$. By Theorem 3.1, the zero-point set $\mathrm{H}_{w^{0}}^{-1}$ of the homotopy equation (3.1) must determine a smooth curve for any given initial point $w^{0} \in \hat{\Omega}^{0}(1) \times R_{++}^{m} \times R^{l}$ as $t \rightarrow 0$, and we can obtain a fixed point of a twice continuous differentiable self-mapping $\phi(x)$ in the general non-convex set $\Omega$. In this paper, we will use standard Euler-Newton predictor-corrector procedure to numerically trace the homotopy path $\Gamma_{w^{0}}$, more details see references, e.g., $[1,17-19,21]$. A detailed description of the implementation on the modified infeasible homotopy method is presented as follows.

\section{Algorithm 4.1.}

Step 1. Initialization.

Set the accuracy parameters $\varepsilon_{1} \geqslant \varepsilon_{2}>0$, given constant $\eta_{i}>0, i=1,2, \cdots, m$, initial point $\chi^{0} \in$ $\hat{\Omega}^{0}(1)$, homotopy parameter $t_{0}=1$, initial step-length $\lambda_{0}>0$, the step-length adjusting parameters $0<\epsilon_{1}<\epsilon_{2}<\epsilon_{3}<1<\epsilon_{4}<\epsilon_{5}$, the maximum number $\bar{N}$ of the corrector steps, the threshold value $\epsilon_{\alpha}$ for the angle between two neighbouring predictor directions and the threshold value $0<\epsilon_{\mathrm{t}}<1$ for starting the end game. Set $k=0$.

Step 2. The first predictor step.

If $k=0$, set $\hat{\lambda}=\lambda_{0}, \varepsilon=\varepsilon_{1}$;

Let $\mathrm{d}^{-1}=(0, \cdots, 0,-1)^{\mathrm{T}} \in \mathrm{R}^{\mathrm{n}+\mathrm{m}+\mathrm{l}+1}$, compute the predictor step $\mathrm{d}$ by

$$
\left(\begin{array}{c}
\operatorname{DH}\left(w^{0}, 1\right) \\
\left(d^{-1}\right)^{\top}
\end{array}\right) d=-d^{-1}
$$

Set $d^{0}=\frac{d}{\|d\|}$; Determine the smallest nonnegative integer $i$ such that

$$
\left(w^{1,0}, t_{1,0}\right)=\left(w^{0}, t_{0}\right)+\epsilon_{3}^{i} \hat{\lambda} d^{0} \in \hat{\Omega}\left(t_{1,0}\right) \times R_{+}^{m} \times R^{l} \times(0,1) \text {, set } \hat{\lambda}=\epsilon_{3}^{i} \hat{\lambda} ; \text { Go to Step } 3 ;
$$

Else. Go to Step 5.

Step 3. The corrector step.

Set $j=0$, repeat;

Compute the Newton step $\hat{\mathrm{d}}$ by solving

$$
\left(\begin{array}{c}
\mathrm{DH}\left(w^{\mathrm{k}+1, j}, \mathrm{t}_{k+1, j}\right) \\
\left(\mathrm{d}^{\mathrm{k}}\right)^{\top}
\end{array}\right) \hat{\mathrm{d}}=\left(\begin{array}{c}
-\mathrm{H}\left(w^{\mathrm{k}+1, j}, \mathrm{t}_{\mathrm{k}+1, j}\right) \\
0
\end{array}\right) .
$$

Determine the smallest nonnegative integer $i$ such that

$$
\begin{aligned}
& \qquad\left(w^{k+1, j+1}, t_{k+1, j+1}\right)=\left(w^{k+1}, t_{k+1}\right)+\epsilon_{3}^{i} \hat{\lambda} \hat{d} \in \hat{\Omega}\left(t_{k+1, j+1}\right) \times R_{+}^{m} \times R^{l} \times(0,1) . \\
& \text { If }\left\|\mathrm{H}\left(w^{k+1, j+1}, t_{k+1, j+1}\right)\right\|_{\infty} \leqslant\left\|H\left(w^{k+1, j}, t_{k+1, j}\right)\right\|_{\infty} \text {, set } j=j+1 ; \\
& \text { Else, set } j=\bar{N},\left(w^{k+1, j}, t_{k+1, j}\right)=\left(w^{k+1,0}, t_{k+1,0}\right) \text {, until }\left\|H\left(w^{k+1, j}, t_{k+1, j}\right)\right\|_{\infty} \leqslant \varepsilon \text { or } j=\bar{N} ;
\end{aligned}
$$

Go to Step 4. 
Step 4. The step-length strategy.

If $j=\bar{N}$ and $\left\|H\left(w^{k+1, j}, t_{k+1, j}\right)\right\|_{\infty}>\varepsilon$, set $\hat{\lambda}=\epsilon_{2} \hat{\lambda},\left(w^{k+1,0}, t_{k+1,0}\right)=\left(w^{k}, t_{k}\right)+\hat{\lambda} d^{k}$;

Go to Step 3;

Else, set $\left(w^{k+1}, t_{k+1}\right)=\left(w^{k+1, j}, t_{k+1, j}\right)$; Adjust the step-length $\hat{\lambda}$ as follows:

$$
\begin{aligned}
& \text { If }\left(d^{k}\right)^{\top} d^{k-1}<\epsilon_{\alpha} \text {, set } \hat{\lambda}=\epsilon_{1} \hat{\lambda} ; \\
& \text { If } j>4 \text {, set } \hat{\lambda}=\epsilon_{2} \hat{\lambda} ; \\
& \text { If } j=2 \text {, set } \hat{\lambda}=\epsilon_{4} \hat{\lambda} ; \\
& \text { If } j<2 \text {, set } \hat{\lambda}=\epsilon_{5} \hat{\lambda} ;
\end{aligned}
$$

If $t_{k+1}<\epsilon_{\mathrm{t}}$, go to Step 6;

If $\left\|\mathrm{H}\left(w^{k+1}, 0\right)\right\|_{\infty} \leqslant \varepsilon_{2}$ and $w^{k+1}$ is feasible, terminate the algorithm. $\tilde{w}=w^{k+1}$ is the computed solution to the equivalent system (2.2). Therefore, the $x$-component $\tilde{x}=x^{k+1}$ is a fixed point.

Set $\varepsilon=\min \left\{t_{k+1}, \varepsilon_{1}\right\}, k=k+1$.

Step 5. The midway predictor step.

Let $d^{k}=\left(\left(w^{k}, t_{k}\right)-\left(w^{k-1}, t_{k-1}\right)\right) /\left\|\left(w^{k}, t_{k}\right)-\left(w^{k-1}, t_{k-1}\right)\right\|$;

Determine the smallest nonnegative integer $i$ such that

$$
\left(w^{k+1,0}, t_{k+1,0}\right)=\left(w^{k}, t_{k}\right)+\epsilon_{3}^{i} \hat{\lambda} d^{k} \in \hat{\Omega}\left(t_{k+1,0}\right) \times R_{+}^{m} \times R^{l} \times(0,1), \text { set } \hat{\lambda}=\epsilon_{3}^{i} \hat{\lambda} ;
$$

Go to Step 3.

Step 6. The end game.

Set $j=0, w^{k+1,0}=w^{k+1}$; Repeat;

Compute the Newton step $d_{\text {end }}$ by solving the equation

$$
\frac{\partial \mathrm{H}}{\partial w}\left(w^{k+1,0}, 0\right) \mathrm{d}_{\mathrm{end}}=-\mathrm{H}\left(w^{k+1, j}, 0\right) ;
$$

Set $w^{k+1, j+1}=w^{k+1, j}+d_{\text {end }}, j=j+1$;

Until, $\left\|\mathrm{H}\left(w^{k+1, j}, 0\right)\right\|_{\infty} \leqslant \varepsilon_{2}$ or $j=\bar{N}$;

If $\left\|\mathrm{H}\left(w^{k+1, j}, 0\right)\right\|_{\infty} \leqslant \varepsilon_{2}$ and $w^{k+1, j}$ is feasible, terminate the algorithm. $\tilde{w}=w^{k+1, j}$ is the computed solution to the equivalent system (2.2). Therefore, the $x$-component $\tilde{x}=x^{k+1, j}$ is a fixed point.

Else, set $\epsilon_{\mathrm{t}}=0.1 \epsilon_{\mathrm{t}}$.

Algorithm 4.1 has been implemented in Matlab and some numerical tests were carried out on a computer running the software Matlab R2014a on Microsoft Windows 7 Professional with Intel(R) $3.40 \mathrm{GHz}$ processor and $8.00 \mathrm{~GB}$ megabytes of memory. And the termination tolerance is set as $\epsilon=10^{-6}$.

For convenience, in the following numerical tests, the shifted constraint functions are constructed as $\hat{g}_{i}(x, t)=g_{i}(x)-t^{\theta} \tau, i=1,2, \cdots, m$, where the constants $\theta$ and $\tau$ are chosen as $\theta=2, \tau=0$ when $g_{i}\left(x^{0}\right)<0$ and $\tau=\max \left\{g_{i}\left(x^{0}\right)\right\}+10$ when $g_{i}\left(x^{0}\right) \nless 0$, respectively. The parameters in the homotopy equation (3.1) are set as $\eta_{i}=10, i=1,2, \cdots, \mathrm{m}$.

The numerical results have been listed in the following tables, CPU denotes the computer time, IT denotes the iteration step which is the summation of the predictor step and the corrector step in the computing process, and $\tilde{x}$ denotes the fixed point of $F(\Omega) \subseteq \Omega$.

Example 4.2. To compute a fixed point of a self-mapping:

$$
\phi(x)=\left(\frac{1}{2} x_{1}+\frac{1}{25} x_{2}, x_{1}^{2}+\frac{1}{4} x_{2}\right)^{\top},
$$


in the non-convex set $\Omega=\left\{\left(x_{1}, x_{2}\right) \in R^{2}:-x_{1}-5 \leqslant 0, x_{1}-5 \leqslant 0, x_{2}-50 \leqslant 0,-x_{1}^{2}+\frac{1}{2} x_{2}=0\right\}$.

In this example, the initial points are chosen as $x_{1}^{0}=(1,1), x_{2}^{0}=(10,-5), x_{3}^{0}=(-15,-10)$ and $x_{4}^{0}=(-30,30)$, which are not interior points in the original feasible set. Hence, the combined homotopy interior point method in $[9,21]$ fails. But, by the homotopy equation (3.1), we can get the unique fixed point $\tilde{x}=(0,0)$ of the self-mapping $\phi(\Omega) \subseteq \Omega$ as $\mathrm{t} \rightarrow 0$. The numerical results are listed in Table 1 .

Table 1: The numerical results of Example 4.2.

\begin{tabular}{cccc}
\hline$x^{(0)}$ & CPU & IT & $\tilde{\chi}$ \\
\hline$(1,1)$ & 0.1404 & 10 & $10^{-7} \times(1.3456,-1.6174)$ \\
$(10,-5)$ & 0.0624 & 19 & $10^{-10} \times(1.2878,-6.8530)$ \\
$(-15,-10)$ & 0.0624 & 21 & $10^{-7} \times(1.5056,-3.8393)$ \\
$(-30,30)$ & 0.1716 & 60 & $10^{-11} \times(0.3587,-2.3512)$ \\
\hline
\end{tabular}

Example 4.3. To compute a fixed point of a self-mapping:

$$
\phi(x)=\left(x_{1},-x_{2}\right)^{\top},
$$

in the non-convex set $\Omega=\left\{\left(x_{1}, x_{2}\right) \in R^{2}:-x_{1}-2 \leqslant 0, x_{2}-5 \leqslant 0,-x_{2}-5 \leqslant 0, x_{1}-x_{2}^{2}+1=0\right\}$.

In this example, the initial points are chosen as $x_{1}^{0}=(-2,-2), x_{2}^{0}=(10,10), x_{3}^{0}=(30,-20)$ and $x_{4}^{0}=(-20,30)$, which are not interior points in the original feasible set. Hence, the combined homotopy interior point method in $[9,20,21]$ fails. But, by the homotopy equation (3.1), we can get the unique fixed point $\tilde{x}=(-1,0)$ of the self-mapping $\phi(\Omega) \subseteq \Omega$ as $t \rightarrow 0$. The numerical results are listed in Table 2 .

Table 2: The numerical results of Example 4.3.

\begin{tabular}{cccc}
\hline$\chi^{(0)}$ & CPU & IT & $\tilde{\chi}$ \\
\hline$(-2,-2)$ & 0.2340 & 21 & $\left(-1,1.5349 \times 10^{-7}\right)$ \\
$(10,10)$ & 0.0468 & 34 & $\left(-1,8.1671 \times 10^{-11}\right)$ \\
$(30,-20)$ & 0.1404 & 54 & $\left(-1,4.0060 \times 10^{-13}\right)$ \\
$(-20,30)$ & 0.0624 & 43 & $\left(-1,1.2826 \times 10^{-8}\right)$ \\
\hline
\end{tabular}

\section{Acknowledgment}

The authors gratefully acknowledge the support of Jilin University of Economics and Finance Funded Foundation (No. 2016Z10, 2016Q31), the Outstanding Youth Foundation of Jilin Provincial Development of Science and Technology (No. 20170520050JH).

\section{References}

[1] E. L. Allgower, K. Georg, Introduction to numerical continuation methods, Reprint of the 1990 edition [SpringerVerlag, Berlin; MR1059455 (92a:65165)], Classics in Applied Mathematics, Society for Industrial and Applied Mathematics (SIAM), Philadelphia, PA, (2003). 4

[2] L. C. Ceng, A. Latif, A. E. Al-Mazrooei, Hybrid viscosity methods for equilibrium problems, variational inequalities, and fixed point problems, Appl. Anal., 95 (2015), 1088-1117. 1

[3] R.-D. Chen, Z.-C. Zhu, Viscosity approximation method for accretive operator in Banach space, Nonlinear Anal., 69 (2008), 1356-1363. 1

[4] S. N. Chow, J. Mallet-Paret, J. A. Yorke, Finding zeroes of maps: homotopy methods that are constructive with probability one, Math. Comp., 32 (1978), 887-899. 1, 2 
[5] X.-N. Fan, F.-R. Gao, T.-T. Xu, An infeasible homotopy method for solving fixed point problems on a general unbounded set, J. Appl. Math. Comput., 47 (2015), 277-289. 1

[6] H.-M. He, S.-Y. Liu, R.-D. Chen, Convergence results of multi-valued nonexpansive mappings in Banach spaces, J. Inequal. Appl., 2014 (2014), 12 pages. 1

[7] R. B. Kellogg, T. Y. Li, J. Yorke, A constructive proof of the Brouwer fixed-point theorem and computational results, SIAM J. Numer. Anal., 13 (1976), 473-483. 1

[8] J. K. Kim, T. M. Tuyen, Regularization proximal point algorithm for finding a common fixed point of a finite family of nonexpansive mappings in Banach spaces, Fixed Point Theory Appl., 2011 (2011), 10 pages. 1

[9] M.-L. Su, Z.-X. Liu, Modified homotopy methods to solve fixed points of self-mapping in a broader class of nonconvex sets, Appl. Numer. Math., 58 (2008), 236-248. 1, 2, 4.2, 4.3

[10] M.-L. Su, X.-H. Qian, Existence of an interior path leading to the solution point of a class of fixed point problems, J. Inequal. Appl., 2015 (2015), 8 pages. 1

[11] M.-L. Su, B. Yu, S.-Y. Shi, A boundary perturbation interior point homotopy method for solving fixed point problems, J. Math. Anal. Appl., 377 (2011), 683-694. 1

[12] Y.-H. Yao, R.-D. Chen, Y.-C. Liou, A unified implicit algorithm for solving the triple-hierarchical constrained optimization problem, Math. Comput. Modelling, 55 (2012), 1506-1515. 1

[13] Y.-H. Yao, R.-D. Chen, J.-C. Yao, Strong convergence and certain control conditions for modified Mann iteration, Nonlinear Anal., 68 (2008), 1687-1693.

[14] Y.-H. Yao, Y.-C. Liou, J.-C. Yao, Convergence theorem for equilibrium problems and fixed point problems of infinite family of nonexpansive mappings, Fixed Point Theory Appl., 2007 (2007), 12 pages.

[15] Y.-H. Yao, Y.-C. Liou, J.-C. Yao, Split common fixed point problem for two quasi-pseudo-contractive operators and its algorithm construction, Fixed Point Theory Appl., 2015 (2015), 19 pages.

[16] Y.-H. Yao, M. Postolache, Y.-C. Liou, Z.-S. Yao, Construction algorithms for a class of monotone variational inequalities, Optim. Lett., 10 (2016), 1519-1528. 1

[17] B. Yu, Z.-H. Lin, Homotopy method for a class of nonconvex Brouwer fixed-point problems, Appl. Math. Comput., 74 (1996), 65-77. 1, 1, 4

[18] Z.-C. Zhu, B. Yu, A modified homotopy method for solving the principal-agent bilevel programming problem, Comput. Appl. Math., 2016 (2016), 1-26.

[19] Z.-C. Zhu, B. Yu, Globally convergent homotopy algorithm for solving the KKT systems to the principal-agent bilevel programming, Optim. Methods Softw., 32 (2017), 69-85. 4

[20] Z.-C. Zhu, B. Yu, Y.-F. Shang, A modified homotopy method for solving nonconvex fixed points problems, Fixed Point Theory, 14 (2013), 531-544. 1, 4.3

[21] Z.-C. Zhu, B. Yu, L. Yang, Globally convergent homotopy method for designing piecewise linear deterministic contractual function, J. Ind. Manag. Optim., 10 (2014), 717-741. 4, 4.2, 4.3

[22] Z.-C. Zhu, Z.-Y. Zhou, Y.-C. Liou, Y.-H. Yao, Y.-C. Xing, A globally convergent method for computing the fixed point of self-mapping on general nonconvex set, J. Nonlinear Convex Anal., 18 (2017), 1067-1078. 1 